Journal of Fluid Mechanics

http://journals.cambridge.org/FLM

Journal of

Additional services for Journal of Fluid Mechanics:

Fluid Mechanics

Email alerts: $\underline{\text { Click here }}$

Subscriptions: $\underline{\text { Click here }}$

Commercial reprints: $\underline{\text { Click here }}$

Terms of use : $\underline{\text { Click here }}$

\title{
The motion of an intense vortex near topography
}

N. ROBB McDONALD

Journal of Fluid Mechanics / Volume 367 / July 1998, pp 359 - 377

DOI: 10.1017/S0022112098001554, Published online: 08 September 2000

Link to this article: http://journals.cambridge.org/abstract_S0022112098001554

How to cite this article:

N. ROBB McDONALD (1998). The motion of an intense vortex near topography. Journal of Fluid Mechanics, 367, pp 359-377 doi:10.1017/S0022112098001554

Request Permissions : $\underline{\text { Click here }}$ 


\title{
The motion of an intense vortex near topography
}

\author{
By N. ROBB MCDONALD \\ Department of Mathematics, University College London, Gower Street, \\ London WC1E 6BT, UK
}

(Received 24 September 1997 and in revised form 10 February 1998)

The initial value problem for the motion of an intense, quasi-geostrophic, equivalentbarotropic, singular vortex near an infinitely long escarpment is studied in three parts. First, for times small compared to the topographic wave timescale the motion of the vortex is analysed by deriving an expression for the secondary circulation caused by the advection of fluid columns across the escarpment. The secondary circulation, in turn, advects the primary vortex and integral expressions are found for its velocity components. Analytical expressions in terms of integrals are found for the vortex drift velocity components. It is found that, initially, cyclones propagate away from the deep water region and anticyclones propagate away from the shallow water region. Asymptotic evaluation of the integrals shows that both cyclones and anticyclones eventually propagate parallel to the escarpment with shallow water on their right at a steady speed which decays exponentially with distance from the escarpment. Secondly, it is shown that for times comparable to, and larger than, the wave timescale, the vortex always resonates with the topographic wave field. The flux of energy in the topographic waves leads to a loss of energy in the vortex and global energy and momentum arguments are used to derive an equation for the distance (or, equivalently, the vortex velocity) of the vortex from the escarpment. It is shown that cyclones, provided they are initially within an $O(1)$ distance (here a unit of distance is dimensionally equivalent to one Rossby radius of deformation) from the escarpment, drift further away from the deep water (i.e. toward higher ambient potential vorticity), possibly crossing the escarpment and accumulate at a distance of $\approx 1.2$ on the shallow side of the escarpment. For distances larger than 1.2 there is essentially no drift of the vortex perpendicular to the escarpment. Anticyclones display similar behaviour except they drift in the opposite direction, i.e. away from the shallow water or toward lower ambient potential vorticity. Third, the method of contour dynamics is used to describe the evolution of the vortex and the interface representing the initial potential vorticity jump between the shallow and deep water regions. The contour dynamic results are in good quantitative agreement with the analytical results.

\section{Introduction}

In the abyssal ocean there are many regions where, at least locally, the background gradient in potential vorticity is dominated by variation in the bathymmetry rather than the ubiquitous planetary curvature, or $\beta$-effect as it is frequently represented as in theoretical models. Such regions may occur, for example, in the vicinity of mid-ocean ridges, such as the mid-Atlantic ridge, or near continental margins. Here, it is expected that the trajectories of abyssal eddies, such as those effecting the dispersal of newly formed bottom water, depend, to leading order, on the local 
topography. Aside from the ocean it is possible that atmospheric eddies such as tropical cyclones, are also influenced by topography such as coastal mountain barriers. As a step towards understanding the role of topography in steering eddies, this paper investigates the initial value problem for a singular, monopole vortex on an $f$ plane with topography using equivalent-barotropic, quasi-geostrophic dynamics. In particular, the topography consists of an infinitely long escarpment and the vortex is assumed to be intense. That is, at least near the vortex, the timescale for the vortex circulation is much shorter than the topographic vortex stretching time so that advection of fluid by the vortex across the escarpment dominates over topographic wave generation. It should be noted however that the dynamics is strictly not quasigeostrophic owing to the three-dimensional nature of the flow at the escarpment. For the small escarpment heights to be considered here such effects are relatively small and are ignored and quasi-geostrophy is assumed to hold everywhere. As well as being important in its own right, the escarpment topography serves as a useful paradigm for the $\beta$-plane problem (and more general continuous background potential vorticity structures). In particular, the direction going from deep to shallow water is analogous to the northward direction on a $\beta$-plane since both directions are toward increasing ambient potential vorticity. Note, however, that in the present problem it is anticipated that the distance of the vortex from the escarpment will be an important parameter in the dynamics but there is no direct analogy to this in the $\beta$-plane problem. This is because in the $\beta$-plane problem the gradient of the ambient potential vorticity is constant, whereas in the present case it is discontinuous.

The theory developed here is similar in spirit to the recent $\beta$-plane studies, which are of particular relevance to the motion of tropical cyclones in the atmosphere, of Reznik (1992), Reznik \& Dewar (1994) and Sutyrin \& Flierl (1994). These studies elucidated the role of the so-called $\beta$-gyres in effecting the movement of vortices. That is, the creation of a secondary dipole circulation as the primary monopole vortex interacts with the background potential vorticity field. The vortex then propagates along the dipole axis. Reznik (1992) considers singular vortices as does the present work, while the works of Reznik \& Dewar (1994) and Sutyrin \& Flierl (1994) consider non-singular, or continuous, vortices. In particular, Reznik \& Dewar (1994) analysed continuously distributed axisymmetric, barotropic vortices and suggested the importance of the effects of planetary wave radiation for large times on, for example, the vortex trajectory. Sutyrin \& Flierl (1994) used a contour dynamical model embedded within a $\beta$-plane to elucidate the development of vortex asymmetries for times small compared to the wave timescale. Also relevant, particularly to ocean eddies, is Grimshaw et al. (1994) who examined, both analytically and numerically, the motion of both weak and intense eddies over a uniformly sloping bottom. Significantly, in their work the fluid motion was barotropic but not quasi-geostrophic so that there is no direct analogy to the $\beta$-plane case. For a weak (small-amplitude) vortex the structure rapidly broke up into topographic shelf waves. For an intense vortex, the initial movement of the eddy was described according to secondary circulations analogous to the $\beta$-gyres. The numerical experiments generally confirmed their theoretical results.

The present work consists of three parts. The first part, described in $\S \S 2$ and 3, is a description of the initial movement of the vortex by secondary circulations established as fluid columns are advected across the escarpment. Second, $\S 4$ investigates times of the order of the topographic vortex stretching time when the vortex is significantly influenced by wave radiation. A description of the far-field, linear wave field is given. Using an approach based on equating the loss of energy of the vortex to the energy 
flux of the far-field waves (or, alternatively, using momentum arguments) a first-order, ordinary differential equation for the evolution of the distance of the vortex from the escarpment is found and subsequently solved numerically. Finally, in $\S 5$, the method of contour dynamics is used to illustrate the motion of the vortex over the whole range of timescales.

\section{Problem formulation}

Quasi-geostrophic motion of fluid on an $f$-plane with topography is governed by the conservation of potential vorticity which in non-dimensional form is

$$
Q_{t}+J[\psi, Q]=0,
$$

where

$$
Q=\nabla^{2} \psi-\psi+\epsilon d
$$

is the potential vorticity. Here $\psi$ is the streamfunction, $t$ is the time, $d(x, y)$ is the topography and $J[f, g]=f_{x} g_{y}-f_{y} g_{x}$ is the Jacobian. The original variables have been made non-dimensional by choosing the lengthscale $L=\left(g^{\prime} H\right)^{1 / 2} / f$ to be the Rossby radius of deformation and the timescale $T$ to be the vortex turnover time, i.e. $T=L / U=H / f \Lambda$. Here $H$ is the average depth of the layer containing the vortex, $f$ is the Coriolis parameter, $g^{\prime}$ the reduced gravity of the active fluid layer, $\Lambda$ is the scale for the vortex amplitude and $U$ is a typical vortex velocity given by geostrophy. It follows that the parameter $\epsilon$ is given by $\epsilon=D / \Lambda=(L / U) /\left((H / D) f^{-1}\right)$, where $D$ is the scale height of the topography, i.e. the ratio of the eddy turnover time to the topographic vortex stretching time. In the following it is assumed that $0<\epsilon \ll 1$, which implies that the effect of topography is relatively small and such a vortex is called intense (cf. Reznik 1992 for the $\beta$-plane case). If, on the other hand, $\epsilon \gg 1$ and $t$ is suitably rescaled, (1) becomes a linear topographic wave equation: the vortex is weak and radiates topographic waves which, in turn, have no leading-order effect on the vortex trajectory. This case is not considered here. Note that the form of (2) implies that equivalent-barotropic dynamics are assumed. In the context of the present work this is an attempt to model the stratification of the abyssal ocean: in particular, a layer of dense fluid underlying a motionless, less dense layer of fluid where the interface between the two layers is free to deform.

In the absence of topography (i.e. $d(x, y) \equiv 0)$ isolated singular vortex solutions to (1) are well known. They are

$$
\Psi=-\frac{\Gamma}{2 \pi} K_{0}\left[\left((x-X)^{2}+(y-Y)^{2}\right)^{1 / 2}\right],
$$

where $K_{0}$ is the modified Bessel function of order zero. Such solutions are obtained from solving

$$
\nabla^{2} \Psi-\Psi=\Gamma \delta(x-X, y-Y) .
$$

The sign of $\Gamma$ gives the sense of the circulation about the vortex: if $\Gamma>0$ the circulation is anticlockwise (cyclonic) or if $\Gamma<0$ the circulation is clockwise (anticyclonic). By integrating (1) over all space and provided $\psi \rightarrow 0$ sufficiently rapidly in the far field, it is straightforward to show that $\mathrm{d} \Gamma / \mathrm{d} t=0$, i.e. $\Gamma$ is constant.

The present work considers the initial value problem for a singular vortex of the form (3) in the presence of non-zero topography. At $t=0$ the vortex has initial location $(X(0), Y(0))=(0, L)$. For $t>0$ the circulation of the vortex advects surrounding fluid particles across isobaths demanding, in turn, an associated change 
in their potential vorticity. This then sets-up secondary circulations which advect the primary vortex. The problem is analogous to determining the path of vortices on a $\beta$-plane (e.g. Reznik 1992; Reznik \& Dewar 1994; Sutyrin \& Flierl 1994), where the secondary circulations are termed $\beta$-gyres. The present work considers another particularly simple choice of topography, namely that of an infinitely long escarpment, i.e. $d(x, y)=\operatorname{sgn}(y)$. The aim is to determine the vortex trajectory, i.e. $X(t)$ and $Y(t)$ for $t \geqslant 0$. Note, in order that the intense vortex assumption be valid, it is required that $\Gamma=O(1)$ and that $L \leqslant O(1)$ throughout its evolution, otherwise the advection of fluid columns across the escarpment by the vortex circulation is weak.

The following analysis is adapted from Reznik (1992) and Grimshaw et al. (1994). It is clear that the magnitude of the secondary circulation is controlled by the degree of topographic vortex stretching, i.e. the secondary circulation scales like $\epsilon$. Thus writing $\psi=\Psi+\epsilon \phi$ and substituting into (1) gives

$$
q_{t}+J[\Psi, q]=-\epsilon J[\phi, q],
$$

where

$$
q=\nabla^{2} \phi-\phi+d .
$$

In a frame of reference moving with the vortex let

$$
x^{\prime}=x-X(t), \quad y^{\prime}=y-Y(t),
$$

where $(X(t), Y(t))$ is the position of the vortex centre. Equation (5) becomes

$$
q_{t}+J^{\prime}[\Psi, q]=q_{x^{\prime}}\left(\frac{\mathrm{d} X}{\mathrm{~d} t}+\epsilon \phi_{y^{\prime}}\right)+q_{y^{\prime}}\left(\frac{\mathrm{d} Y}{\mathrm{~d} t}-\epsilon \phi_{x^{\prime}}\right) .
$$

Here it is understood that $d=d(y)=d\left(y^{\prime}+Y(t)\right)$. Since the vortex velocity is determined by the $\phi$ field it follows thats

$$
\frac{\mathrm{d} X}{\mathrm{~d} t}=-\left.\epsilon \phi_{y^{\prime}}\right|_{x^{\prime}=y^{\prime}=0}, \quad \frac{\mathrm{d} Y}{\mathrm{~d} t}=\left.\epsilon \phi_{x^{\prime}}\right|_{x^{\prime}=y^{\prime}=0} .
$$

Thus $\mathrm{d} X / \mathrm{d} t$ and $\mathrm{d} Y / \mathrm{d} t$ are both $O(\epsilon)$. Letting $\mathrm{d} X / \mathrm{d} t=\epsilon u$ and $\mathrm{d} Y / \mathrm{d} t=\epsilon v$, (8) becomes

$$
q_{t}+J^{\prime}[\Psi, q]=\epsilon q_{x^{\prime}}\left(u+\phi_{y^{\prime}}\right)+\epsilon q_{y^{\prime}}\left(v-\phi_{x^{\prime}}\right) .
$$

Notice that the right-hand side of (10) becomes identically equal to zero at the vortex centre. To leading order (10) becomes

$$
q_{t}+b(r) q_{\theta}=0,
$$

where $r^{2}=x^{\prime 2}+y^{\prime 2}, \theta=\arctan \left(y^{\prime} / x^{\prime}\right)$ and $b(r)=\Psi_{r} / r=\Gamma K_{1}(r) / 2 \pi r$. Equation (11) has solution

$$
q=q[\theta-b(r) t, r] .
$$

At $t=0, q=d\left(y^{\prime}+Y(0)\right)=d(r \sin \theta+L)$ and so, in general,

$$
q=d[r \sin (\theta-b t)+L] .
$$

Substituting (13) into (6) gives

$$
\nabla^{2} \phi-\phi=d[r \sin (\theta-b t)+L]-d[r \sin \theta+Y(t)] .
$$

Since

$$
Y(t)=L+\epsilon \int_{0}^{t} v(t) \mathrm{d} t
$$


it follows that for $t \ll \epsilon^{-1}, Y(t)=L+O(\epsilon)$. Neglecting terms of $O(\epsilon)$ and for the particular choice of escarpment topography, the secondary circulation $\phi$ is determined by solution of

$$
\nabla^{2} \phi-\phi=\operatorname{sgn}[r \sin (\theta-b t)+L]-\operatorname{sgn}[r \sin \theta+L] .
$$

Once $\phi$ is known the vortex velocity, and hence trajectory, can be deduced from $(9 a, b)$.

3. Solution for $0 \leqslant t \ll \epsilon^{-1}$

In (16) put $\phi=\phi_{1}+\phi_{2}$ where

$$
\nabla^{2} \phi_{1}-\phi_{1}=-\operatorname{sgn}\left[y^{\prime}+L\right], \quad \nabla^{2} \phi_{2}-\phi_{2}=\operatorname{sgn}[r \sin (\theta-b t)+L] .
$$

Using the Green's function method, the solution for $\phi_{1}$ is

$$
\phi_{1}=\frac{1}{2 \pi} \int_{-\infty}^{\infty} \int_{-\infty}^{\infty} \operatorname{sgn}[\zeta+L] K_{0}\left[\left(\left(x^{\prime}-\xi\right)^{2}+\left(y^{\prime}-\zeta\right)^{2}\right)^{1 / 2}\right] \mathrm{d} \xi \mathrm{d} \zeta .
$$

Calculating the velocity components of the vortex associated with $\phi_{1}$ given by (18) according to $u_{1}=-\left.\phi_{1 y^{\prime}}\right|_{x^{\prime}=y^{\prime}=0}$ and $v_{1}=\left.\phi_{1 x^{\prime}}\right|_{x^{\prime}=y^{\prime}=0}(\mathrm{cf} 9 a, b)$ gives

$$
u_{1}=-\exp (-|L|), \quad v_{1}=0 .
$$

The expression for $\phi_{2}$, also obtained using the Green's function method, working this time in polar coordinates to accommodate the $(r, \theta)$ dependence, is

$\phi_{2}=-\frac{1}{2 \pi} \int_{0}^{\infty} \int_{0}^{2 \pi} \operatorname{sgn}[\rho \sin (\alpha-b t)+L] K_{0}\left[\left(r^{2}+\rho^{2}-2 r \rho \cos (\theta-\alpha)\right)^{1 / 2}\right] \rho \mathrm{d} \alpha \mathrm{d} \rho$.

The vortex velocity components associated with $\phi_{2}$ are given by

$$
u_{2}=-\phi_{2 r} \sin \theta-\frac{1}{r} \phi_{2 \theta} \cos \theta, \quad v_{2}=\phi_{2 r} \cos \theta-\frac{1}{r} \phi_{2 \theta} \sin \theta
$$

where the above expressions are to be evaluated at $r=0$. Use of $(20)$ in $(21 a, b)$ yields

$$
\begin{aligned}
u_{2} & =\frac{1}{2 \pi} \int_{0}^{\infty} \int_{0}^{2 \pi} \operatorname{sgn}[\rho \sin (\alpha-b t)+L] K_{1}(\rho) \rho \sin \alpha \mathrm{d} \alpha \mathrm{d} \rho, \\
& =\frac{1}{2 \pi} \int_{L}^{\infty} \int_{0}^{2 \pi} \operatorname{sgn}[\sin (\alpha-b t)+L / \rho] K_{1}(\rho) \rho \sin \alpha \mathrm{d} \alpha \mathrm{d} \rho,
\end{aligned}
$$

and

$$
\begin{aligned}
v_{2} & =-\frac{1}{2 \pi} \int_{0}^{\infty} \int_{0}^{2 \pi} \operatorname{sgn}[\rho \sin (\alpha-b t)+L] K_{1}(\rho) \rho \cos \alpha \mathrm{d} \alpha \mathrm{d} \rho, \\
& =-\frac{1}{2 \pi} \int_{L}^{\infty} \int_{0}^{2 \pi} \operatorname{sgn}[\sin (\alpha-b t)+L / \rho] K_{1}(\rho) \rho \sin \alpha \mathrm{d} \alpha \mathrm{d} \rho,
\end{aligned}
$$

where $-1 \leqslant L / \rho \leqslant 1$ in the final expressions for $u$ and $v$ in $(22 a, b)$. 
Consider the integral $\left(I_{1}\right.$ say) in $(22 a)$ with respect to $\alpha$, i.e.

$$
\begin{aligned}
I_{1} & =\int_{0}^{2 \pi} \operatorname{sgn}[\sin (\alpha-b t)+L / \rho] \sin \alpha \mathrm{d} \alpha, \\
& =\int_{-b t}^{2 \pi-b t} \operatorname{sgn}[\sin \alpha+L / \rho] \sin (\alpha+b t) \mathrm{d} \alpha, \\
& =\cos b t \int_{-b t}^{2 \pi-b t} \operatorname{sgn}[\sin \alpha+L / \rho] \sin \alpha \mathrm{d} \alpha+\sin b t \int_{-b t}^{2 \pi-b t} \operatorname{sgn}[\sin \alpha+L / \rho] \cos \alpha \mathrm{d} \alpha .
\end{aligned}
$$

Denote $\alpha_{c 1,2}$ to be the critical values of $\alpha$ where the sgn-function changes sign (generally there are two such values in the interval; $-b t \leqslant \alpha \leqslant 2 \pi-b t)$, i.e. $\alpha_{c 1,2}=\arcsin (-L / \rho)$. By dividing the integrals in (23) into three intervals according to whether the sgnfunction is positive or negative it is straightforward to show that

$$
\int_{-b t}^{2 \pi-b t} \operatorname{sgn}[\sin (\alpha)+L / \rho] \sin \alpha \mathrm{d} \alpha=4\left|\cos \alpha_{c}\right|=\frac{4}{\rho}\left(\rho^{2}-L^{2}\right)^{1 / 2}
$$

and

$$
\int_{-b t}^{2 \pi-b t} \operatorname{sgn}[\sin (\alpha)+L / \rho] \cos \alpha \mathrm{d} \alpha=0
$$

Therefore from $(19 a, b)$ and $(22 a, b)$ it follows that the vortex velocity components are given by

$$
\begin{aligned}
u=u_{1}+u_{2} & =-\exp (-|L|)+\frac{2}{\pi} \int_{L}^{\infty} \cos (b t) K_{1}(r)\left(r^{2}-L^{2}\right)^{1 / 2} \mathrm{~d} r \\
& =\frac{2}{\pi} \int_{L}^{\infty}(\cos b t-1) K_{1}(r)\left(r^{2}-L^{2}\right)^{1 / 2} \mathrm{~d} r, \\
v=v_{1}+v_{2} & =\frac{2}{\pi} \int_{L}^{\infty} \sin (b t) K_{1}(r)\left(r^{2}-L^{2}\right)^{1 / 2} \mathrm{~d} r
\end{aligned}
$$

Note that both $u$ and $v$ vanish at $t=0$.

Asymptotic results for $u$ and $v$, analogous to Reznik (1992), are derived from $(25 a, b)$ in Appendix A and are quoted here. As $t \rightarrow 0$, and provided $L \neq 0$,

$$
v \sim A \Gamma t / 2 \pi, \quad u \sim-B \Gamma^{2} t^{2} / 4 \pi^{2},
$$

where $A$ and $B$ are positive constants which depend on $L$. For the special case $L=0$,

$$
v \sim \operatorname{sgn}(\Gamma)\left(\frac{|\Gamma| t}{\pi^{2}}\right)^{1 / 2}, \quad u \sim-\left(\frac{|\Gamma| t}{\pi^{2}}\right)^{1 / 2} .
$$

Since $\operatorname{sgn} v=\operatorname{sgn} \Gamma$ and $\operatorname{sgn} u=-1$ in $(26 a, b)$ and $(27 a, b)$, it is evident that for small times cyclones move toward negative $x$ and positive $y$, while anticyclones move also toward negative $x$ but negative $y$. A sketch illustrating the physical mechanism for this behaviour is shown in figure 1. Cyclones push fluid columns across the escarpment and conservation of potential vorticity demands that the fluid columns produce relative vorticity and circulation in the sense shown in figure 1(a)(i). In particular, a fluid column going from deep to shallow water gains negative relative vorticity and produces clockwise circulation. On the other hand, a fluid column going from shallow to deep water gains positive relative vorticity and therefore anticlockwise circulation. These secondary circulations then advect the vortex centre in the direction indicated by the arrows. The net result is an initial northward drift. The situation for 
(a)

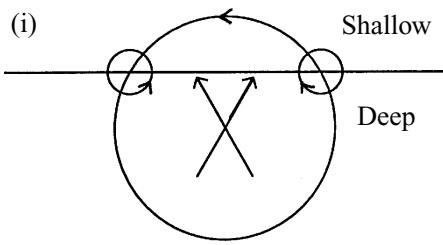

(ii)

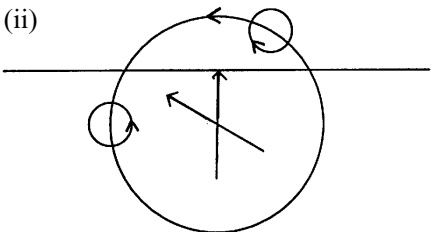

(b)

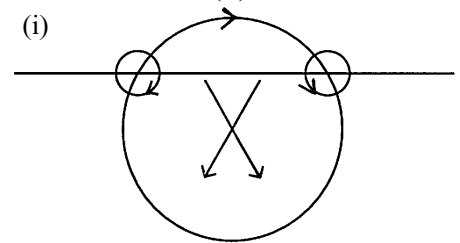

(ii)

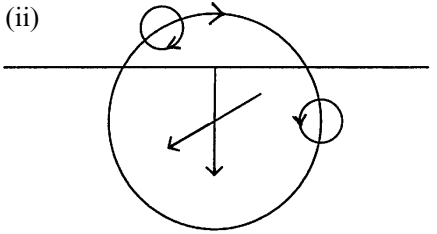

FIGURE 1. Sketch showing the advection of fluid by the vortex and consequent generation of secondary circulations at the escarpment represented by the horizontal line. Here $(a)$ is a cyclone and $(b)$ an anticyclone. The initial secondary circulations are shown in (i) and the direction of the velocities at the vortex centre due to the secondary circulations are shown. The position of these secondary circulations are shown at some time later in (ii), demonstrating that both cyclones and anticyclones both have a 'westward' component to their drift.

an anticyclone is shown in figure 1(b)(i): here the vortex responds to the secondary circulations by moving toward decreasing $y$. At some time later the primary vortex advects these initial secondary circulations (more secondary circulations, of course, appear at the escarpment each time fluid is forced to cross the step) to the position indicated in figure 1(a)(ii) and figure 1(b)(ii). Again the direction of the velocity vector due to the secondary circulation is shown at the vortex centre. It is evident that both cyclones and anticyclones have a component of drift toward decreasing $x$.

Identifying decreasing $x$ as being westward and increasing $y$ as being northward (as will be done from time to time in the present work), such behaviour is precisely that of vortex motion on a $\beta$-plane, i.e cyclones initially propagate northwest and anticyclones initially propagate southwest (see, for example, Reznik 1992). Such an analogy is, of course, expected given the escarpment topography used here: the shallow fluid for $y>0$ has relatively large ambient potential vorticity whereas the deeper fluid for $y<0$ has relatively small ambient potential vorticity. Thus the background potential vorticity gradient increases in the same sense as it does on a $\beta$-plane. Contrasting the $t^{1 / 2}$ behaviour of the vortex velocity when $L=0$, with the $t$ behaviour when $L \neq 0$, it follows that the initial vortex velocity is much greater for the special case when $L=0$. The cause for this is evident by referring to figure 1 . In particular, the closer the vortex centre to the escarpment, the greater the effect of the secondary circulations at the vortex centre since these circulations, which initially occur on the escarpment, are therefore closer to the vortex centre. Moreover the closer the vortex centre to the escarpment the more fluid that is swept across the escarpment. This implies a larger distribution of secondary circulations at the escarpment, which also contributes to a greater velocity induced at the vortex centre. Further, note that $u$ is of the same order as $v$ for the special case $L=0$. This is because of the Bessel function structure of the primary vortex. In particular, in the limit that the distance from the primary vortex tends to zero, the angular velocity of the primary vortex becomes infinite at the escarpment (since $L=0$ ). This implies that the secondary circulations generated infinitesimally close to the primary vortex are effectively swept 
around instantaneously, i.e. the effect illustrated in figure 1 in going from (a)(i) to (a)(ii), say, is instantaneous. Thus $u$ and $v$ are of the same order for small $t$. Note that this effect is purely a consequence of the choice of singular primary vortex and would not occur for, say, a vortex with a continuous distribution of vorticity. This behaviour is independent of whether the vortex is a cyclone or an anticyclone. The net result is that the initial motion is precisely northwest for a cyclone and precisely southwest for an anticyclone. On the other hand, for $L \neq 0, u / v \rightarrow 0$ as $t \rightarrow 0$ and so the immediate movement of the vortex is northward for a cyclone and southward for an anticyclone.

For $t \gg 1$ (but still less than $\epsilon^{-1}$ ), from Appendix A

$$
u=-\exp (-|L|)+O\left(\frac{\log ^{2} t}{t}\right), \quad v=\operatorname{sgn}(\Gamma) O\left(\frac{\log ^{2} t}{t}\right) .
$$

Again the $\beta$-plane analogy holds: cyclones move northwestward and anticyclones move southwestward. There is a slow decay in $v$, and $u$ also slowly approaches the steady speed $\exp (-|L|)$. Note that this speed is independent of the sign and magnitude of $\Gamma$. The steady propagation speed is maximal at $L=0$ and decays exponentially with increasing distance from the escarpment. This result is obtained using an alternative method in Appendix B, namely by seeking steadily translating solutions to (1). The purely westward drift of the vortex at large times may also be explained in terms of secondary circulations. First note that because of the uniform depth either side of the escarpment, once a fluid column has crossed the escarpment its relative vorticity is conserved. Thus fluid on the shallow side of the escarpment can only ever acquire negative relative vorticity and on the deep side it can only ever acquire positive relative vorticity. This is true for both cyclones and anticyclones. This results in a dipole-like structure with a continuous distribution of negative relative vorticity for $y>0$ and a continuous distribution of positive relative vorticity for $y<0$. Note that the dipole is not symmetric because the circulation of the primary vortex for $L \neq 0$ is not symmetric about the escarpment. The dipolar structure is also evident in the steady part of the secondary circulation $\phi_{1}$, given by (18), from which it is straightforward to show that $\phi_{1}>0$ for $y>0$ and $\phi_{1}<0$ for $y<0$, i.e. $\phi_{1}$ changes sign at the escarpment. The mutual advection of the dipole is such that it drives the vortex west. This is precisely the same mechanism by which the $\beta$-gyres act to produce the westward drift of $\beta$-plane vortices.

Complementing these asymptotic results, figure $2(a-c)$ show plots of $v, u$ and the vortex trajectory obtained from FFT and numerical integration of $(25 a, b)$. In each part of figure two curves are shown corresponding to the cases $L=0$ and $L=1$. In each case $\Gamma=+2 \pi$, i.e. a cyclone. Figure $2(a)$ shows the initial rapid, increase in $v$ with time and the subsequent slow decay. Observe that $v \geqslant 0$ for all $t$ implying that the vortex never moves south. Figure $2(b)$ shows the approach to the steady-state velocities $u \rightarrow-1$ for $L=0$ and $u \rightarrow-\mathrm{e}^{-1} \approx-0.37$ for $L=1$. Figure $2(c)$ shows the vortex trajectory up to $t=20$. As expected the vortex propagates both further north and west in the $L=0$ case since it is closer to the escarpment and thus 'feels' the background potential vorticity gradient more. Notice also, consistent with the small time asymptotic results, that the trajectory for the $L=1$ case is initially much steeper (i.e. more northward) than the $L=0$ case. For the case $\Gamma=-2 \pi$, an anticyclone, (not shown) the results are identical except for $v$ changing sign and therefore implying a general southwest propagation.

Perhaps the most interesting point is that both cyclones and anticyclones approach a final velocity $\mathrm{d} X / \mathrm{d} t$ given by $-\epsilon \exp (-|L|)$ and thus $-\epsilon \leqslant \mathrm{d} X / \mathrm{d} t \leqslant 0$. It is well known 

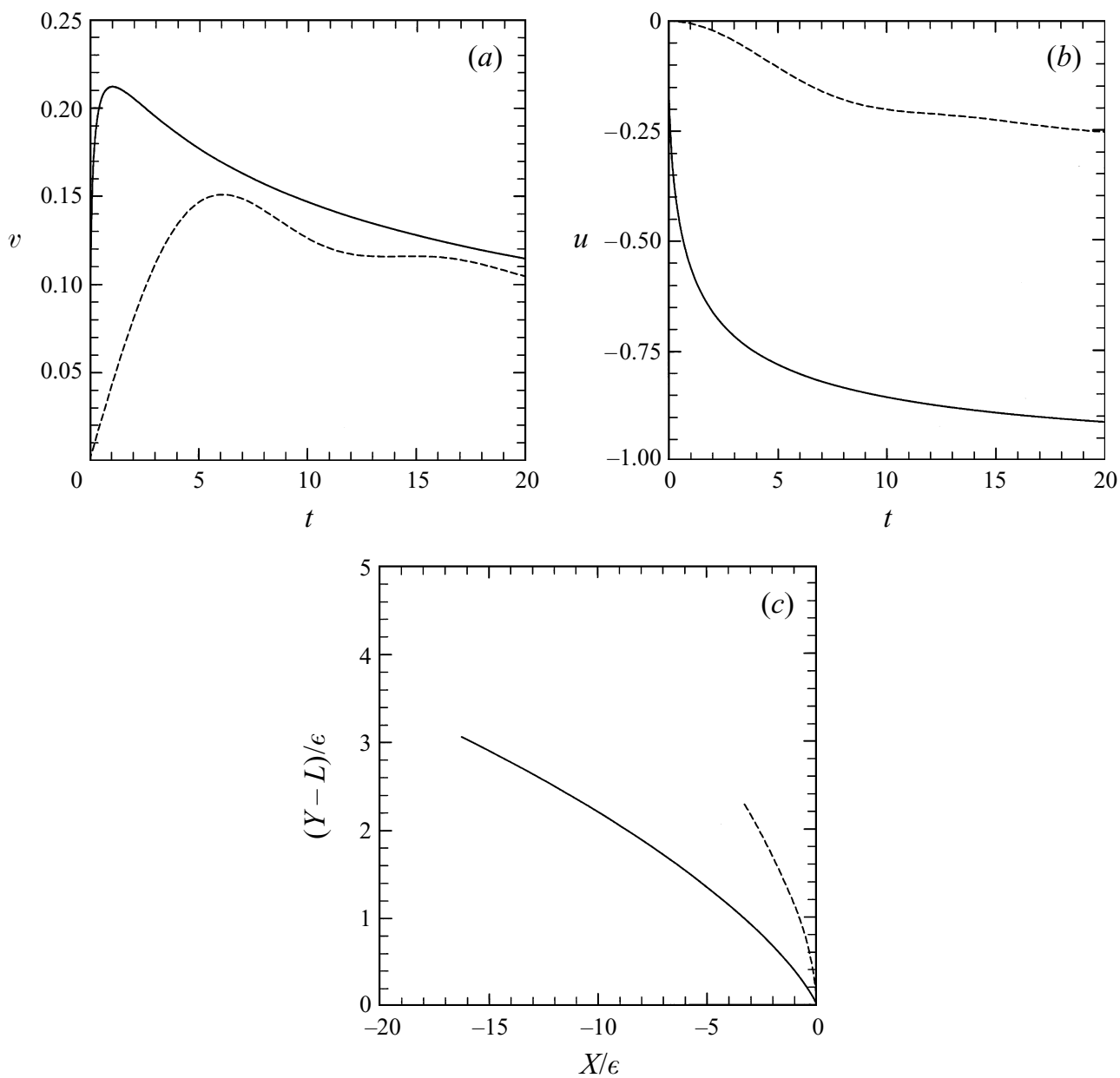

FIGURE 2. Properties of the vortex propagation with $\Gamma=+2 \pi$ and $L=0$ (solid line) and $L=1$ dashed line. (a) The velocity component $v$ for $0 \leqslant t \leqslant 20,(b)$ the velocity component $u$ for $0 \leqslant t \leqslant 20$ and $(c)$ the vortex trajectory (i.e. the displacement $(X, Y-L) / \epsilon)$ up to $t=20$.

that the linearized version of (1) with escarpment topography has free topographic wave solutions with phase velocities $c_{p}$ in the range $-\epsilon \leqslant c_{p} \leqslant 0$ (see e.g. Johnson \& Davey 1989; McDonald 1996). An immediate consequence is that, whatever the initial value of $L$, at large times the vortex must resonate with the topographic wave field, i.e. it moves with a velocity which matches a possible topographic wave phase speed. Such waves have group velocity $c_{g} \neq c_{p}$ (McDonald 1996) and so energy must be lost through topographic wave radiation. The response of the vortex to this energy loss is the subject of the next section.

\section{Large time behaviour}

For times comparable to, and larger than, $\epsilon^{-1}$ the effects of topographic wave radiation must be taken into account. To analyse their effect, the slow timescale $T=\epsilon t$ is introduced into (1). Moreover, sufficiently far from the vortex $\Psi=O(\epsilon)$ and letting $\phi \equiv \eta$, (5) becomes

$$
\left(\nabla^{2} \eta-\eta\right)_{T}+J\left[\eta, \nabla^{2} \eta-\eta\right]+\eta_{x} d_{y}=-J\left[\Psi, \nabla^{2} \eta-\eta\right]-\Psi_{x} d_{y} .
$$


This is a forced topographic wave equation for $\eta$. Formal solution of this equation is inhibited by the presence of the nonlinear Jacobian term involving $\eta$ on the left-hand side. However it is anticipated that much can be learned by ignoring the Jacobian terms in (29). Certainly, ignoring these terms is valid for $\Psi \ll O(1)$ since this implies $\eta \ll 1$, as the magnitude of $\eta$ is determined by the forcing $-\Psi_{x} d_{y}$. This will always be the case at some finite distance from the vortex owing to the exponential-like decay of (3) with distance from the vortex centre.

The solution procedure taken here is as follows: for $t \geqslant \epsilon^{-1}$ the steady zonally propagating vortex of the previous section is taken to be the forcing in (29) which is solved (without the Jacobian terms) to determine the far-field disturbance $\eta$. The energy flux in the far-field topographic waves is then used to calculate the energy loss in the interior vortex solution. An alternative procedure based on momentum conservation, giving identical results, is also presented. Note that since $\Gamma$ is a constant (see §2) the only parameter that can vary on this slow timescale as a result of topographic wave radiation is the speed of the vortex which is directly related to its distance $L$ from the escarpment, i.e. $L=L(T)$. The aim of this section is to calculate $L(T)$. It is perhaps worth emphasizing that the vortex cannot change shape in response to the radiative loss of energy since the Bessel function structure is fixed for a singular vortex. A more general formulation, not considered here, would consist of a continuous distribution of singular vortices of the Bessel function type weighted by a structure function, i.e. a Green's function approach. It would then be possible that such vortices rearrange themselves in response to the radiation to a lower energy configuration which would, possibly, manifest itself as a change in shape of the vortex.

In a frame of reference moving with velocity $c=-\exp (-|L|)$ in the $x$-direction and ignoring the Jacobian terms (29) becomes

$$
\left(\nabla^{2} \eta-\eta\right)_{T}-c\left(\nabla^{2} \eta-\eta\right)_{x}+\eta_{x} d_{y}=-\Psi_{x} d_{y},
$$

where

$$
\Psi=-\frac{\Gamma}{2 \pi} K_{0}\left[\left(x^{2}+(y-L)^{2}\right)\right],
$$

and $d=\operatorname{sgn} y$. Here it is understood that $L$ is a slowly varying function of time (on the $T$ timescale) and both $\eta$ and $\Psi$ are $O(\epsilon)$. Further, it will be shown a posteriori that $\mathrm{d} L / \mathrm{d} T=O(\epsilon)$ so that, to leading order, the time derivatives of $L$, and hence $c$, can be ignored. Equation (30) is similar to that analysed by Johnson \& Davey (1989) and McDonald (1996) where details of the solution technique can be found. The solution of (30) subject to $\eta=0$ at $T=0$ is merely quoted here: it is

$$
\eta=\frac{1}{2 \pi} \int_{-\infty}^{\infty} A(k, T) \mathrm{e}^{-\left(k^{2}+1\right)^{1 / 2}|y|} \mathrm{e}^{\mathrm{i} k x} \mathrm{~d} k,
$$

where

$$
A(k, T)=\frac{\Gamma}{2\left(1+k^{2}\right)} \frac{\mathrm{e}^{-\left(k^{2}+1\right)^{1 / 2}|L|}}{c+\left(k^{2}+1\right)^{-1 / 2}}\left(1-\exp \left[\mathrm{i} k T\left(c+\left(k^{2}+1\right)^{-1 / 2}\right)\right]\right) .
$$

As in Johnson \& Davey (1989) and McDonald (1996), the solution (33) consists of a steady, localized disturbance and an unsteady term representing propagating topographic wave modes. It should be noted that for $y \neq 0$ the solution (32) satisfies $\nabla^{2} \eta-\eta=0$ which is further justification for ignoring the Jacobian terms in (29). In 
this moving frame of reference the topographic waves have dispersion relation

$$
\omega(k)=-c k-\frac{k}{\left(k^{2}+1\right)^{1 / 2}},
$$

and hence the phase and group velocities are

$$
c_{p}=-c-\frac{1}{\left(k^{2}+1\right)^{1 / 2}}, \quad c_{g}=-c-\frac{1}{\left(k^{2}+1\right)^{3 / 2}} .
$$

As demonstrated in McDonald (1996) the large time response is dominated by waves of zero phase velocity, i.e. near the poles of the steady part of (33). This is the resonance condition and since $-1 \leqslant c<0$ (recall $c=-\exp (-|L|)$ ) resonance will always occur (since singularities in the form of simple poles will always be apparent in the steady part of (33)). Note also that for $c_{p}=0$

$$
c_{g}=-c+c^{3}>0,
$$

for $-1 \leqslant c<0$. That is, energy is radiated toward positive values of $x$. The special case $c=-1$ for which $c_{g}=0$ is not considered here, since, after the initial adjustment described in the previous section, $L \neq 0$, implying $c \neq-1$.

For large times and $-1<c<0$, a residue calculation gives (cf. McDonald 1996)

$$
\eta=-\frac{\Gamma}{\gamma c} \mathrm{e}^{(|L|+|y|) / c} \sin \gamma x\left[H\left(x-c_{g} T\right)-H(x)\right],
$$

where $\gamma=\left(-1+1 / c^{2}\right)^{1 / 2}$. The energy density in the waves is

$$
\rho=\frac{1}{2}\left(\eta_{x}^{2}+\eta_{y}^{2}+\eta^{2}\right)=\frac{\Gamma^{2}}{2 \gamma^{2} c^{4}} \mathrm{e}^{2(|L|+|y|) / c},
$$

where an average over one wavelength has been taken. Integrating the energy density from $y=-\infty$ to $y=+\infty$ and multiplying by the group velocity (36), the energy flux $F$ of the topographic waves is found:

$$
F=\frac{\Gamma^{2}}{2} \mathrm{e}^{2|L| / c} .
$$

The next task is to relate this energy flux to the change of energy of the vortex. The energy is defined using an appropriate equivalent-barotropic generalisation of Batchelor (1967, p. 530) (see also Young 1985), namely

$$
E=-\frac{1}{2} \iint \psi\left(\nabla^{2} \psi-\psi\right) \mathrm{d} A^{\prime},
$$

where the integral is over all space. As in Batchelor, self-contributions of singular vortices to the energy integral are ignored so that the leading-order contribution to the energy depends on the relative position of the vorticity fields $\phi$ and $\Psi$. In the present case, to leading order, the energy is

$$
\begin{aligned}
E & =-\frac{\epsilon}{2} \iint\left(\nabla^{2} \phi-\phi\right) \Psi \mathrm{d} A^{\prime}-\frac{\epsilon}{2} \iint\left(\nabla^{2} \Psi-\Psi\right) \phi \mathrm{d} A^{\prime} \\
& =\frac{\epsilon \Gamma}{4 \pi} \iint\left(\nabla^{2} \phi-\phi\right) K_{0}\left[\left(x^{\prime 2}+y^{\prime 2}\right)^{1 / 2}\right] \mathrm{d} A^{\prime}-\frac{\epsilon \Gamma}{2} \phi(0,0),
\end{aligned}
$$

where $\Psi$ and $\phi$ refer to quantities calculated in $\S \S 2$ and 3. In order to calculate this energy observe that, as demonstrated in the previous section, at large times the 
contribution of the oscillatory terms in (16) may be ignored. Thus

$$
\nabla^{2} \phi-\phi=\operatorname{sgn} L-\operatorname{sgn}\left(y^{\prime}+L\right)
$$

and therefore

$$
\phi(0,0)=-\frac{1}{2 \pi} \int_{-\infty}^{\infty} \int_{-\infty}^{\infty}[\operatorname{sgn} L-\operatorname{sgn}(\zeta+L)] K_{0}\left[\left(\xi^{2}+\zeta^{2}\right)^{1 / 2}\right] \mathrm{d} \xi \mathrm{d} \zeta .
$$

Substituting (42) and (43) into (41) gives

$$
\begin{aligned}
E & =\frac{\epsilon \Gamma}{2 \pi} \int_{-\infty}^{\infty} \int_{-\infty}^{\infty}[\operatorname{sgn} L-\operatorname{sgn}(\zeta+L)] K_{0}\left[\left(\xi^{2}+\zeta^{2}\right)^{1 / 2}\right] \mathrm{d} \xi \mathrm{d} \zeta \\
& =\epsilon \operatorname{sgn}(L) \Gamma \mathrm{e}^{-|L|} .
\end{aligned}
$$

Next the relation $\mathrm{d} E / \mathrm{d} T=-\epsilon^{2} F$, relating the leading-order rate of change of energy to the total flux of energy carried by the topographic waves, is used. Here the factor $\epsilon^{2}$ comes from the fact that $\eta=O(\epsilon)$ in the topographic wave field. This gives

$$
\frac{\mathrm{d} L}{\mathrm{~d} T}=\frac{\epsilon \Gamma}{2} \mathrm{e}^{|L|(1+2 / c)}=\frac{\epsilon \Gamma}{2} \exp [|L|(1-2 \exp |L|)] .
$$

Alternatively, (45) can be derived by equating the rate of change of momentum of the vortex with the flux of momentum in the topographic waves. In particular, using the definition of Young (1985) (cf. Batchelor 1967), the leading-order $x$-momentum is

$$
M_{x}=-\iint y\left(\nabla^{2} \Psi-\Psi\right) \mathrm{d} A^{\prime}=-\Gamma L .
$$

The flux of momentum in the topographic wavetrain is given by $F / c$ (an $O(\epsilon)$ quantity), i.e. the energy flux divided by the phase velocity (see e.g. Whitham 1974). Taking the time derivative of (46) and equating it to the flux of momentum $F / c$ yields the result (45).

Equation (45) is a first-order, autonomous differential equation for $L=L(T)$. Note that $\mathrm{d} L / \mathrm{d} T=O(\epsilon)$ and therefore $\mathrm{d} c / \mathrm{d} T=O(\epsilon)$, justifying the assumption that $L$ and $c$ are slowly varying on the $T$ timescale. Two other features are immediately obvious. First, cyclones have $\operatorname{sgn} \Gamma>0$ and so $\mathrm{d} L / \mathrm{d} t>0$, i.e. they drift northward in response to the topographic wave radiation. Similarly, for anticyclones, $\mathrm{d} L / \mathrm{d} T<0$ and therefore they drift southward. Secondly, referring to figure 3 where a plot of $\exp [|L|(1-2 \exp |L|)]$ (i.e. the right-hand side of (45)) as a function of $|L|$ is shown, the maximum rate of change of $L$ occurs when $L=0$. Moreover, for $|L| \geqslant 1.2$, the rate of change of $|L|$ is negligible and it is expected that vortices sufficiently far from the escarpment remain at constant distance from the escarpment. The critical distance of about 1.2 is due to the sensitivity of the energy flux, given by (39), to the distance from the escarpment $|L|$. There are two reasons for this sensitivity. First, as $|L|$ increases, (37) implies that the amplitude of the topographic waves decreases exponentially. Secondly, as $L$ increases, the drift speed $c$ decreases exponentially. In turn (35a) (with $c_{p}=0$, the resonance condition) implies that the resonant wavenumber $k$ increases. From (33), as $k$ increases the amplitude of the waves also decreases exponentially. Hence an increase in $|L|$ causes the amplitude to decrease exponentially via two different mechanisms. The energy flux, being proportional to the square of the wave amplitude, is therefore very sensitive (in an exponential way) to changes in $|L|$.

These features are reinforced by numerical solution of

$$
\frac{\mathrm{d} L}{\mathrm{~d} \tau}=\operatorname{sgn}(\Gamma) \exp [|L|(1-2 \exp |L|)],
$$




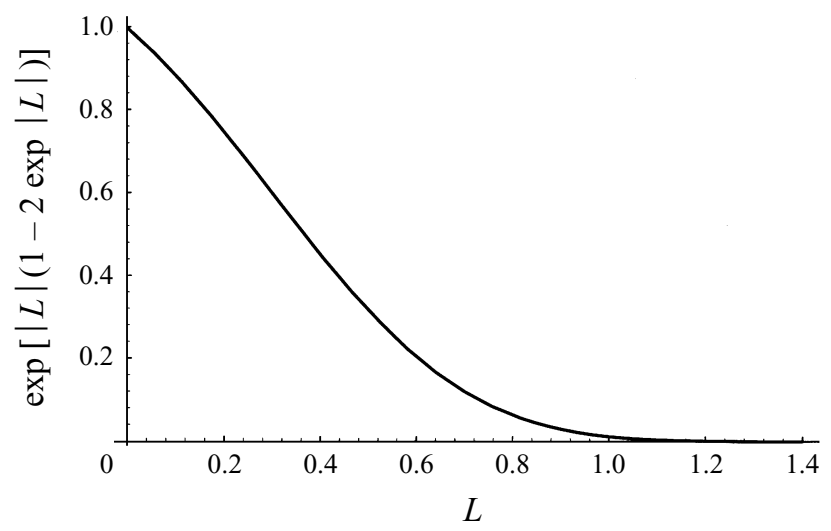

FIgURE 3. The quantity $\exp [|L|(1-2 \exp |L|)]$ as a function of $|L|$.

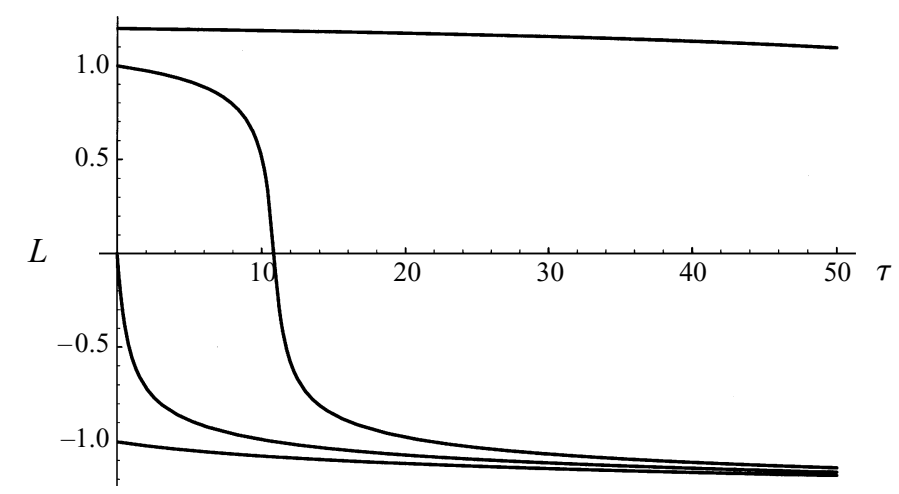

FIGURE 4. Numerical solution of (47) showing $L$ as a function of $\tau$ for four different initial conditions: $L=1.2,1,0$ and -1 . Here $\Gamma<0$, i.e. an anticyclone.

where $\tau$ is a suitably scaled time variable. Figure 4 shows the behaviour of an anticyclone $(\operatorname{sgn} \Gamma<0)$ for various values of $L(0)$, namely $L(0)=1.2,1,0$ and -1 . The tendency of the anticyclone to propagate south is clearly evident. For $L \geqslant 1.2$ the vortex effectively remains at constant $L$ owing to the (super)exponentially small rate of change of $L$. For $L(0)=1$ and $L(0)=0$ the vortex rapidly moves south before asymptoting to $L \approx-1.2$. For $L \leqslant-1.2$ the rate of change of $L$ is again (super)exponentially small and the vortex effectively remains at a fixed distance from the escarpment, i.e. $L$ is constant. The situation is the same for cyclones except that they drift north and if $-1.2<L(0)<1.2$ the cyclones drift toward $L \approx 1.2$. Summarizing, if $L(0)=\mathrm{O}(1)$ then the 'latitudes' $L= \pm 1.2$ appear to be of critical importance with cyclones accumulating at $L \approx 1.2$ and anticyclones at $L \approx-1.2$ after very long times. For $|L|=1.2$ the vortex still 'feels' the escarpment but the energy flux in the radiated topographic wave field given by (39) is is (super)exponentially small.

\section{Contour dynamics}

The choice of an infinitely long escarpment for the topography means that the method of contour dynamics is ideally suited to studying this problem. The contour initially lies along $y=0$, the interface between the deep and shallow water regions, and after the vortex is switched on it evolves according to the conservation of potential 
vorticity. The velocity field associated with the deflected contour (i.e. the secondary circulation) is used to advect the vortex. The algorithm used is based on Dritschel (1988). The time-stepping is done by a fourth-order Runge-Kutta scheme and nodes are redistributed along each contour after each time-step according to the local curvature. The contour dynamics algorithm integrates the full nonlinear potential vorticity equation (1) and is valid for arbitrary deformations of the contour from its initial position. In the following numerical experiments the extent of the contour along $y=0$ is sufficiently long that end-effects may be ignored. More precisely, the computational domain is at least 30 units in the $x$-direction in all the results reported here. The main purpose of the contour dynamics is to provide a check on the analytical results valid in the limit $\epsilon \rightarrow 0$. An exploration of the wave-vortex behaviour for order-one and large $\epsilon$, will be left for subsequent work.

It is worth highlighting a subtle difference between the analytical approach of the previous section and the contour dynamics method. In the analytical results, at $t=0$, the topography was switched on near a pre-existing vortex. In contrast, in the contour dynamics approach a vortex flow is switched on near a pre-existing potential vorticity contour lying along the escarpment. It shall be assumed that this difference in the initialization of the problem makes no difference in the subsequent evolution of the wave-vortex interaction. This assumption is vindicated, as will be seen in this section, when the analytical results for the vortex trajectory are compared to those obtained by the contour dynamics method.

To see how the contour responds to the presence of the vortex its evolution is plotted in figure 5 for times $t=2$ and $t=10$ for a vortex with $\Gamma=\pi, \epsilon=0.05$ and initial location $(0,0.5)$. The anticlockwise circulation of the cyclone wraps-up the contour, an effect which becomes even more pronounced as time increases. This wrapping-up effect, a large deformation of the potential vorticity contour, is expected for the intense vortex regime and would not necessarily occur for larger values of $\epsilon$ where wave radiation could be sufficiently strong to prevent the wrapping-up of the contour. Note that, in agreement to the theory of $\S 3$, the cyclonic vortex has moved upward and to the left. Figure 6 shows the evolution of the contour for the case of an anticyclone. The contour now wraps up according to the clockwise circulation of the vortex. In this case the displacement of the vortex is downward and to the left.

The trajectory of a cyclone with $\Gamma=\pi$ starting at $(0,0.5)$ for $0 \leqslant t \leqslant 10$ for values of $\epsilon=0.1,0.05$ and 0.001 , is shown in figure 7. The displacement of the vortex divided by $\epsilon$ is plotted so that the trajectory may be compared to that obtained analytically by integrating the velocity components $(25 a, b)$. It is clear that as $\epsilon$ decreases the trajectories obtained by the contour dynamics method approach that of the theoretical result. This is expected since the trajectory obtained from $(25 a, b)$ corresponds to the limit $\epsilon \rightarrow 0$. In fact for $\epsilon=0.001$ (not shown) the contour dynamic trajectory and the analytical result virtually coincide on the scale of this plot. Another feature to note is that the contour dynamic simulations give trajectories that are slightly more displaced in the $x$-direction but slightly more displaced in the $y$-direction and that this feature becomes more pronounced as $\epsilon$ increases. This is consistent with the fact that if a vortex is displaced further from the escarpment (i.e. greater $|y|$ ), its along-escarpment velocity decreases since it decays exponentially with $|y|$ and thus it is displaced less in the $x$-direction. Trajectories for anticyclones and also from other starting positions were also investigated and all also showed good quantitative agreement with the analytical results particularly as $\epsilon \rightarrow 0$.

Figure 8 shows the differing trajectories for a cyclone and anticyclone with equal magnitudes (i.e. $\Gamma= \pm \pi$ ) both starting at $(0,0.5)$ for $0 \leqslant t \leqslant 140$ and $\epsilon=0.1$. 
The motion of an intense vortex near topography
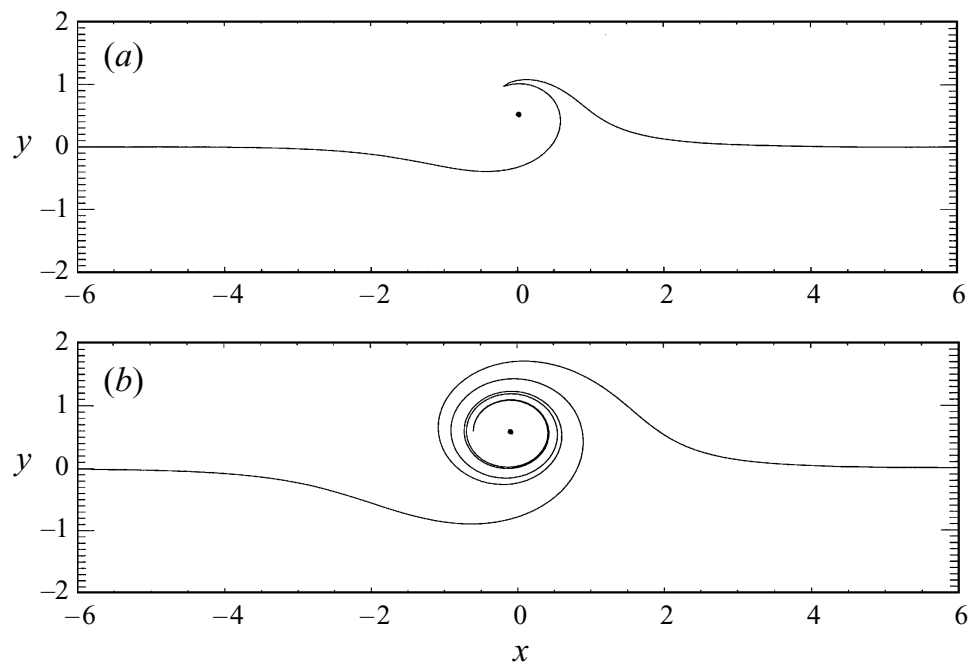

FIGURE 5. Evolution of the potential vorticity interface at times $(a) t=2$ and $(b) t=10$ for a cyclone with $\Gamma=\pi, \epsilon=0.05$ and $L=0.5$. The position of the vortex is indicated by the dot.
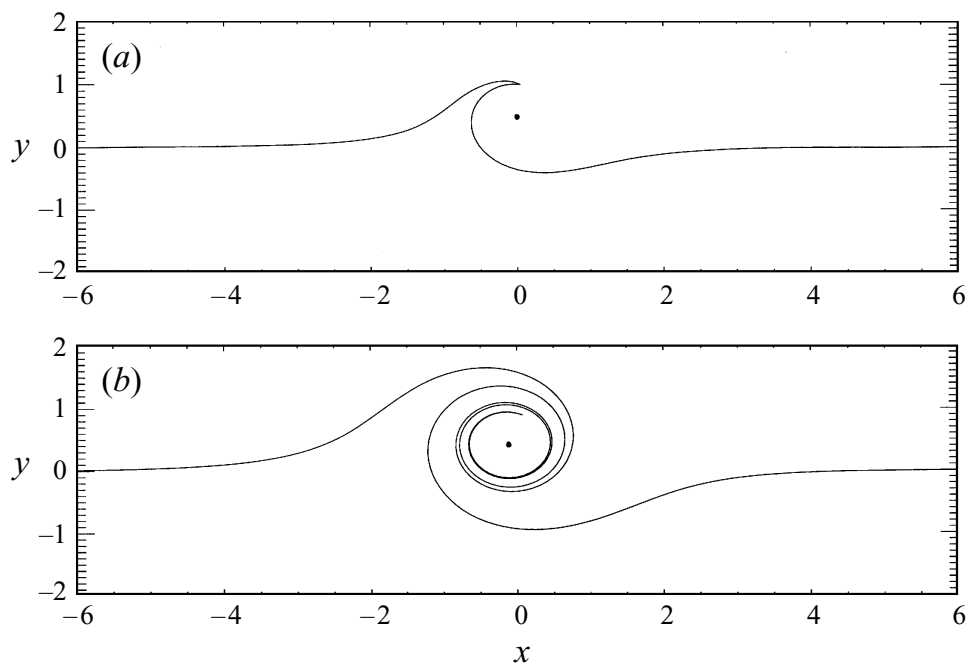

FIGURE 6. As in figure 5 but for an anticyclone with $\Gamma=-\pi$.

The cyclone, which moves toward positive $y$ is displaced less distance in the $x$ direction than the anticyclone. This is due to the fact that, for most of its journey, the anticyclone is closer to the escarpment at $y=0$ and therefore it has a velocity in the $x$-direction greater in magnitude than the cyclone (cf. $(28 a)$ ). Note also that the anticyclone crosses the escarpment and continues to drift toward larger values of $-y$. Towards the end of the integration both of the trajectories appear to have levelled off, reaching a constant value of $y$. It is expected that wave radiation, discussed in the previous section, continues to influence the vortex trajectories leading to slow drift away from the escarpment. However this effect is difficult to observe in the contour-dynamic model since the drift velocity is very slow and, for the choice of $\epsilon=0.1$, would require the model to be run to for several hundred more units in time 


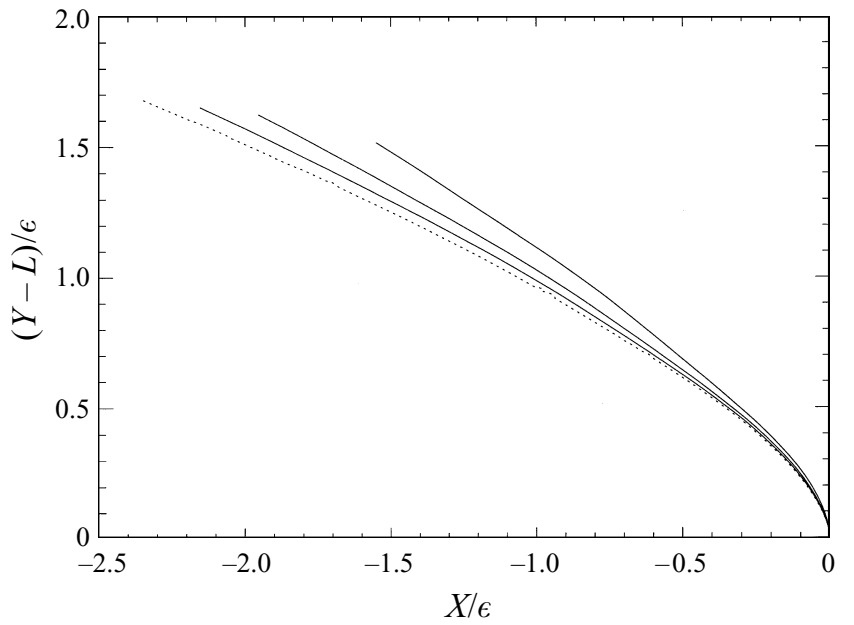

FIGURE 7. A comparison of vortex trajectories for a cyclone with $\Gamma=\pi$ and $L=0.5$ for $0 \leqslant t \leqslant 10$ for various values of $\epsilon$. The rightmost solid curve corresponds to $\epsilon=0.1$, the middle solid curve to $\epsilon=0.05$ and the leftmost solid curve to $\epsilon=0.01$. The dashed curve corresponds to the trajectory obtained from the analytical result $(25 a, b)$.

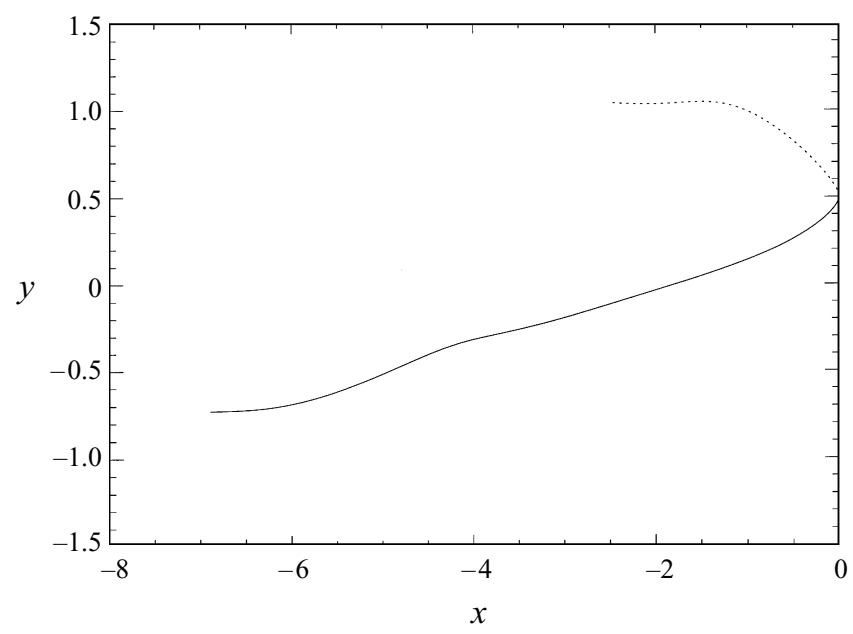

FiguRE 8. Vortex trajectories for a cyclone ( $\Gamma=\pi$, dashed line) and anticyclone $(\Gamma=-\pi$, solid line) both starting at $L=0.5$ for $0 \leqslant t \leqslant 140$ and $\epsilon=0.1$.

to observe any significant drift. The integration rapidly becomes computationally expensive since, with every time step, the contour becomes increasingly 'wrapped up' (cf. figures 5 and 6) meaning that more points need to be added to the contour.

\section{Conclusions}

An example of wave-vortex interaction has been studied in the limit that the vortex is intense. That is, the timescale for the vortex circulation is much smaller than the timescale associated with the wave propagation. Here the restoring force for the wave propagation is provided by the piecewise uniform potential vorticity due to the escarpment topography. 
The initial motion of the vortex is toward regions of lower potential vorticity for cyclones and away from regions of lower potential vorticity for anticyclones. This behaviour is a result of secondary circulations demanded by potential vorticity conservation as fluid columns are swept across the escarpment topography by the vortex circulation. As time increases the velocity of the vortex at right angles to the potential vorticity gradient decreases and a steady motion along the escarpment, with shallow water on the right for both cyclones and anticyclones, ensues. The velocity of this steady motion of the vortex decays exponentially with distance from the escarpment.

After the initial adjustment to a purely along-escarpment motion, both cyclones and anticyclones resonate with the topographic wave field. By linearizing the equations of motion in the far field, an expression for the radiated topographic wave field was obtained. Both momentum- and energy-based arguments were used to establish that the vortex drifts at right angles to the escarpment in response to the wave radiation. Being a singular vortex this is the only way in which a singular vortex can respond (i.e. it cannot change shape as may occur, for example, with a continuous distribution of singular vortices). In particular, cyclones drift toward regions of higher ambient potential vorticity while anticyclones drift in the opposite direction. At a distance equal to, or greater than, about 1.2 Rossby radii from the escarpment the drift becomes negligible and, for practical purposes, the vortex remains at a fixed distance from the escarpment. The direction of the radiation-induced drift is in common with the well-known meridional drift of $\beta$-plane eddies, namely that cyclones drift north and anticyclones drift south.

It is interesting to note that while both anticyclones and cyclones drift toward negative $x$ in this intense regime, a weak vortex will drift in accordance with the direction of its image. This is because in the weak limit, radiative effects dominate at the escarpment and after the initial transients have radiated away, no fluid crosses the escarpment. The escarpment therefore acts like a wall in that the normal velocity component vanishes (cf. McDonald 1993). Thus in this case anticyclones and cyclones propagate in opposite directions according to their image. This suggests interesting behaviour, in particular asymmetry between cyclone and anticyclone trajectories, as the parameter $\epsilon$ is varied from small to large values. This will be explored elsewhere.

It is also worth noting that the use of singular vortices is somewhat restrictive in that it precludes other modes of response of the vortex to radiative energy loss. In particular, a distributed vortex has the ability to change shape and size as it loses energy. Hence the drift in the $y$-direction may not be the only effect on a distributed vortex due to radiative energy loss. The issue of the response of a distributed vortex requires further study.

I thank Ted Johnson for useful discussions on aspects of this work. I also thank the referees for their valuable comments which have led to improvements in the paper.

\section{Appendix A. Asymptotic results}

Asymptotic results for the integral expressions of the vortex velocity components $u$ and $v(25 a, b)$ are derived in this section.

\section{A.1. Behaviour as $t \rightarrow 0$}

There are two cases to consider: If $L \neq 0$ then the $t \rightarrow 0$ behaviour is obtained by making the approximations $\sin b t \approx \Gamma K_{1}(r) t / 2 \pi r$ in $(25 b)$ and $1-\cos b t \approx \Gamma^{2} K_{1}^{2}(r) t^{2} / 4 \pi^{2} r^{2}$ 
in $(25 a)$ and noting the subsequent integrals with respect to $r$ converge. This yields

$$
v=\frac{A \Gamma t}{2 \pi}, \quad u=-\frac{B \Gamma^{2} t^{2}}{4 \pi^{2}}
$$

where $A$ and $B$ are integral expressions, which depend on $L$, whose integrands are positive definite over the range $[L, \infty)$. Hence $A$ and $B$ are positive constants.

This procedure does not work for $L=0$ since the resulting integrals with respect to $r$ are non-convergent. Instead, consider $v=v_{1}+v_{2}$ where

$$
v_{1}=\frac{2}{\pi} \int_{0}^{r_{1}} \sin (b t) r K_{1}(r) \mathrm{d} r, \quad v_{2}=\frac{2}{\pi} \int_{r_{1}}^{\infty} \sin (b t) r K_{1}(r) \mathrm{d} r .
$$

Here $r_{1}$ is small but fixed. Again for similar reasons as outlined above the second integral $v_{2}$ is $O(t)$ as $t \rightarrow 0$. For small $r, K_{1}(r) \sim 1 / r$ and so introducing the new variable $\xi=|\Gamma| / r^{2}, v_{1}$ can be written as

$$
v_{1}=\frac{(|\Gamma| / 2 \pi)^{1 / 2} \operatorname{sgn} \Gamma}{\pi} \int_{\xi}^{\infty} \frac{\sin (\xi t)}{\xi^{3 / 2}} \mathrm{~d} \xi
$$

where $\xi_{1}=|\Gamma| / r_{1}^{2}$. Integration by parts and another change of variable yields

$$
v_{1}=\frac{(|\Gamma| / 2 \pi)^{1 / 2} \operatorname{sgn} \Gamma}{\pi}\left\{\frac{2 \sin \alpha}{\xi_{1}^{1 / 2}}+2 t^{1 / 2} \int_{\alpha}^{\infty} \frac{\cos z}{z^{1 / 2}} \mathrm{~d} z\right\},
$$

where $\alpha=\xi_{1}$ t. As $t \rightarrow 0$ the integral term in (A 4) is dominant since

$$
\int_{0}^{\infty} \frac{\cos z}{z^{1 / 2}} \mathrm{~d} z=\left(\frac{\pi}{2}\right)^{1 / 2}
$$

and hence $v \sim \operatorname{sgn} \Gamma\left(|\Gamma| t / \pi^{2}\right)^{1 / 2}$. A similar calculation also yields $u \sim-\left(|\Gamma| t / \pi^{2}\right)^{1 / 2}$ as $t \rightarrow 0$.

\section{A.2. Behaviour as $t \rightarrow \infty$}

Consider $v=v_{1}+v_{2}$ where

$v_{1}=\frac{2}{\pi} \int_{L}^{r_{1}} \sin (b t)\left(r^{2}-L^{2}\right)^{1 / 2} K_{1}(r) \mathrm{d} r, \quad v_{2}=\frac{2}{\pi} \int_{r_{1}}^{\infty} \sin (b t)\left(r^{2}-L^{2}\right)^{1 / 2} K_{1}(r) \mathrm{d} r . \quad(\mathrm{A} 6 a, b)$

Here $r_{1}$ is large but fixed. Note that $b^{\prime}(r)=-\Gamma K_{2}(r) / 2 \pi r$ and so $b^{\prime}(r) \neq 0$ on the interval $L \leqslant r \leqslant r_{1}$. Moreover, $\left(r^{2}-L^{2}\right)^{1 / 2} K_{1}(r)$ is integrable on this interval. The conditions required for application of the Riemann-Lebesgue lemma thus apply and therefore

$$
v_{1}=O\left(t^{-1}\right)
$$

as $t \rightarrow \infty$. For $r_{1}$ sufficiently large $\left(r^{2}-L^{2}\right)^{1 / 2} \approx r$ and $K_{n}(r) \approx \exp (-r) / r^{1 / 2}$ for $n=1$ and 2 . Hence

$$
\begin{aligned}
v_{2} & \approx \frac{2}{\pi} \int_{r_{1}}^{\infty} \sin (b t) r K_{1}(r) \mathrm{d} r=\frac{4}{\Gamma} \int_{0}^{\xi_{1}} \sin (\xi t) \log ^{2}(\xi) \mathrm{d} \xi \\
& =\frac{4}{\Gamma t} \int_{0}^{\xi_{1} t} \sin z \log ^{2}\left(\frac{z}{t}\right) \mathrm{d} z,
\end{aligned}
$$

where $\xi_{1}=|\Gamma| K_{1}\left(r_{1}\right) / r_{1}$. Integration by parts may be used to establish that the integral in (A 8) has time-dependent leading-order behaviour $\log ^{2} t$ as $t \rightarrow \infty$. Thus, 
to leading order,

$$
v=\frac{4}{\Gamma t} \log ^{2} t
$$

A similar result holds for the oscillatory part of $u$ as $t \rightarrow \infty$ implying

$$
u=-\exp (-|L|)+O\left(\frac{\log ^{2} t}{t}\right) \text {. }
$$

Similar results were obtained by Reznik (1992) for a singular vortex on a $\beta$-plane.

\section{Appendix B. Alternative derivation of final vortex speed}

Assuming that the vortex is propagating steadily in the $x$-direction with velocity $\epsilon c$, in a frame of reference $\left(x^{\prime}, y^{\prime}\right)$ centred on the vortex (1) becomes

$$
-\epsilon c Q_{x^{\prime}}+J^{\prime}[\psi, Q]+\epsilon \psi_{x^{\prime}} d_{y^{\prime}}=0,
$$

for the case when $d=d\left(y^{\prime}\right)$. Multiplying by $x^{\prime}$ and integrating over all space and using the fact that the vortex is isolated yields

$$
c=\iint \psi d_{y^{\prime}} \mathrm{d} A^{\prime} / \iint Q \mathrm{~d} A^{\prime} .
$$

For the case $Q=\Gamma \delta\left(x^{\prime}, y^{\prime}\right)$ then $\psi=-(\Gamma / 2 \pi) K_{0}(r)$ (cf. (3) and (4)) and for $\mathrm{d}\left(y^{\prime}\right)=\operatorname{sgn}\left(y^{\prime}-L\right)$ then $\mathrm{d}_{y}^{\prime}=2 \delta\left(y^{\prime}-L\right)$. Thus

$$
c=-\frac{1}{\pi} \int_{-\infty}^{\infty} K_{0}\left[\left(x^{\prime 2}+L^{2}\right)^{1 / 2}\right] \mathrm{d} x^{\prime}=-\exp (-|L|),
$$

in agreement with the asymptotic result in the text.

\section{REFERENCES}

Batchelor, G. K. 1967 An Introduction to Fluid Dynamics. Cambridge University Press.

Dritschel, D. G. 1988 Contour surgery: a topological reconnection scheme for extended integrations using contour dynamics. J. Comput. Phys. 77, 240-266.

Grimshaw, R. H. J., Broutman, D., He, Xinyu \& Sun, Pei. 1994 Analytical and numerical study of a barotropic eddy on a topographic slope. J. Phys. Oceanogr. 24, 1587-1607.

Johnson, E. R. \& DAVEY, M. K. 1989 Free-surface adjustment and topographic waves in coastal currents. J. Fluid Mech. 219, 273-289.

McDonald, N. R. 1993 Topographic dispersal of bottom water. J. Phys. Oceanogr. 23, 954-969.

McDonald, N. R. 1996 Topographic wave generation and modon decay. Geophys. Astrophys. Fluid Dyn. 83, 51-77.

Reznik, G. M. 1992 Dynamics of singular vortices on a beta-plane. J. Fluid Mech. 240, 405-432.

ReZnik, G. M. \& Dewar, W. K. 1994 An analytical theory of distributed axisymmetric barotropic vortices on the $\beta$-plane. J. Fluid Mech. 269, 301-321.

Sutyrin, G. G. \& Flierl, G. R. 1994 Intense vortex motion on the beta plane: development of the beta gyres. J. Atmos. Sci. 51, 773-790.

Whitham, G. B. 1974 Linear and Nonlinear Waves. Wiley.

YouNG, W. R. 1985 Some interactions between small numbers of baroclinic geostrophic vortices. Geophys. Astrophys. Fluid Dyn. 33, 35-61. 